\title{
Augmented Reality
}

My attention was recently drawn to a remarkable
seven-minute TED video by Louie Schwartzberg
that reinforced for me the power of technology to
adapt to the limitations of our human perceptions.

With the aid of technology, often digital in nature and often involving some serious computation, we can perceive that which is too fast, too slow, too big, too small, too diverse, and too high or low (as in frequency). As Schwartzberg's video illustrates, we can use time-lapse photography to watch processes too slow to perceive or highspeed photography to make visible that which is too fast for the human eye to see. We can downshift or upshift frequencies to make things audible that we would otherwise not detect: the low-frequency communication of elephants $^{\mathrm{b}}$ and the high frequencies generated by bats and pest-control devices. We can shift or detect highenergy and high-frequency photons, such as X-rays, and make them visible to the human eye. We can take images in ultraviolet or infrared that our eyes cannot see but our instruments can, and thus make them visible.

Anyone who has watched a timelapse film of flowers opening or mushrooms growing or vines climbing can appreciate how dramatically the timelapse images help us appreciate and understand processes that take place so slowly that we do not see them as dynamic. I recall visiting a rain forest in Irian Jaya (the western half of Papua New Guinea) where our guide explained the long, slow battle between the trees and the climbing vines that, ultimately, throttled the trees over a period of years. I recall when my son,

a https://www.youtube.com/watch?v=FiZqn6fV-4Y b https://www.youtube.com/watch?v=YfHO6bM6v8k
David, suggested a 100-year project to photograph, in time-lapse, a forest's vivid story. It would be quite an interesting experience to watch the slow, titanic battles for control of the upper canopy and the survival and regeneration of the ground-hugging brush over the course of decades. It would be a technical challenge to ensure the equipment stayed functional, but one could use radio transmission to capture the images as long as the cameras were in operating condition. Similar tactics have been used to observe, on a continuous basis, areas not friendly to human habitation such as winters at the poles.

The rise of interest in "big data" has spurred a concurrent interest in visualization of collections of digital information, looking for patterns more easily recognized by humans than by computer algorithms. Creation of overlays of multiple data sources on Google Earth, correlated as to time and geographic location, also have served as an organized way to visualize and experience information we could not naturally observe with our human senses. Similar methods have brought visibility to the distribution of dark matter in the universe by inferring its existence and presence through its gravitational effects.

As our computational tools become more and more powerful, we can anticipate that our growing knowledge of the mechanics of our world will allow us to use simulation to visualize, understand, and even design processes that we could only crudely imagine before. The 2013 Nobel Prize for Chemistry went to Martin Karplus, Michael Levitt, and Arieh Warshel "for the development of multiscale models for complex chemical systems." This is computational chemistry at its best and it shows how far we have come with tools that depend upon significant computing power available in this second decade of the $21^{\text {st }}$ century. Indeed, we hear, more and more, of computational physics, biology, linguistics, exegesis, and comparative literature as fields well outside the traditional numerical analysis and programming disciplines typically associated with computer science. Computation has become an infrastructure for the pursuit of research in a growing number of fields of science and technology, including sociology, economics, and behavioral studies.

One can only speculate what further accumulation of digitized data, computational power, storage, and models will bring in the future. The vast troves of data coming from the Large Hadron Collider, the Hubble, and future James Webb telescopes (among others), and the NSF National Ecological Observation Network (NEON) program ${ }^{c}$ will be the sources for visualization, correlation, and analysis in the years ahead. Whoever thinks computer science is boring has not been paying attention!

\footnotetext{
c http://www.nsf.gov/funding/pgm_summ.jsp and http://www.neoninc.org/
}

Vinton G. Cerf is vice president and Chief Internet Evangelist at Google. He served as ACM president from 2012-2014.

Copyright held by author. 